Original Article

\title{
Comparison of changes in the mobility of the pelvic floor muscle on during the abdominal drawing-in maneuver, maximal expiration, and pelvic floor muscle maximal contraction
}

\author{
Halim Jung, PT, MS ${ }^{1)}$, Sangwoo Jung, PT, MS ${ }^{1)}$, Sunghee Joo, PT, MS ${ }^{1)}$, Changho Song, PT, PhD ${ }^{1 *}$ \\ 1) Department of Physical Therapy, Sahmyook University: 815 Hwarangro, Nowon-gu, Seoul 139-742, \\ Republic of Korea
}

\begin{abstract}
Purpose] The purpose of this study was to compare changes in the mobility of the pelvic floor muscle during the abdominal drawing-in maneuver, maximal expiration, and pelvic floor muscle maximal contraction. [Subjects] Thirty healthy adults participated in this study (15 men and 15 women). [Methods] All participants performed a bridge exercise and abdominal curl-up during the abdominal drawing-in maneuver, maximal expiration, and pelvic floor muscle maximal contraction. Pelvic floor mobility was evaluated as the distance from the bladder base using ultrasound. [Results] According to exercise method, bridge exercise and abdominal curl-ups led to significantly different pelvic floor mobility. The pelvic floor muscle was elevated during the abdominal drawingin maneuver and descended during maximal expiration. Finally, pelvic floor muscle mobility was greater during abdominal curl-up than during the bridge exercise. [Conclusion] According to these results, the abdominal drawingin maneuver induced pelvic floor muscle contraction, and pelvic floor muscle contraction was greater during the abdominal curl-up than during the bridge exercise.

Key words: Pelvic floor muscle, Abdominal drawing-in maneuver, Ultrasound imaging
\end{abstract}

(This article was submitted Oct. 15, 2015, and was accepted Oct. 31, 2015)

\section{INTRODUCTION}

Pelvic floor muscles support the floor and abdominal cavity of the pelvis and have the dual function of regulating urination and urinary pelvic stability. Pelvic floor muscle dysfunction is associated with dysuria and pelvic pain ${ }^{1)}$. Women with back pain and men with pelvic pain show decreased function of the pelvic floor muscles ${ }^{2,3}$. For effective training of the pelvic floor muscles, simultaneous contraction of the muscles around the trunk should be considered, and the part for the breathing associated with features of the trunk muscle must also be considered ${ }^{1)}$. Mohseni-Bandpei et al. ${ }^{4)}$ reported that the pelvic floor muscle training should be combined with trunk stabilization exercises.

To promote trunk stability, abdominal curl-up and bridging exercises are widely used for clinical applications. The abdominal curl-up is an exercise that increases abdominal muscle activity, is used to strengthen the abdomen ${ }^{5}$, and is a trunk stabilization exercise in which gross and local muscles are used in an appropriate ratio ${ }^{6}$.

Interventions to induce simultaneous contraction of the deep muscles during trunk stabilization exercise have been proposed. The abdominal drawing-in maneuver is an exercise in which the transversus abdominis muscle contracts alone while the internal and external abdominal obliques do not move. This exercise is a method of drawing the abdomen toward the spine at the end of normal expiration ${ }^{7)}$. Another intervention is the maximum aerobic method of requiring the maximum contraction of the abdominal muscles by a special method of expiration at the end of normal expiration. This method increases

*Corresponding author. Changho Song (E-mail: chsong@syu.ac.kr)

(C2016 The Society of Physical Therapy Science. Published by IPEC Inc.

This is an open-access article distributed under the terms of the Creative Commons Attribution Non-Commercial No Derivatives (by-nc-nd) License $<$ http://creativecommons.org/licenses/by-nc-nd/4.0/>. 
the muscle thickness of the transverse abdominis and internal oblique muscles and decreases the thickness of the external oblique muscle ${ }^{8)}$. Some studies have reported that this method induces activity of the transverse abdominis muscle better than the abdominal drawing-in maneuver ${ }^{9}$. However, there have been only a few studies on the effect of abdominal drawing-in and maximal expiration on functions of the pelvic floor muscle.

Ultrasound is a new method for measuring functions of the pelvic floor muscle and is noninvasive, safe, and useful for visualization. In particular, a strength of this method is its easy application, since individuals can be assessed while clothed ${ }^{10)}$. Abdominal ultrasound is an objective tool for measuring the strength and functions of the pelvic floor muscle, having significant correlations with measurements obtained by manual assessment of the vagina, measurements obtained with a vaginal pressure meter, or perineal ultrasound ${ }^{11-14)}$.

Therefore, this study attempted to investigate the effects of abdominal drawing-in and maximal expiration, a method of inducing contraction of the deep muscle, during the abdominal curl-up and bridging exercises used as pelvic stabilization exercises. This study thus compared functions of the pelvic floor muscle.

\section{SUBJECTS AND METHODS}

This study was conducted on 30 healthy men and women aged 20-30 years. The inclusion criteria for the participants were as follows: no neurological disorders or muscle lesions of the pelvis and no experienced urogenital disorders within the preceding 6 months. Before the experiment, an explanation about the purpose and procedure of this study was provided to the participants. All participants provided informed consent prior to participation. The study protocol was approved by the Institution Review Board of Sahmyook University. Thirty adults, including 15 women and 15 men, who had agreed to participate in the study and met our inclusion criteria were selected as participants. The selected participants were asked to learn how to perform the abdominal drawing-in maneuver and pelvic floor muscle maximal contraction before the experiment while they were video-monitored with ultrasound equipment ${ }^{15,16)}$.

Participants were instructed to check the location of the bladder and then to perform the abdominal drawing-in maneuver, maximum expiration, and pelvic floor muscle maximum contraction. The movement distance of the lower bladder was measured 3 times using an ultrasound measurement tool for each movement per exercise ${ }^{17)}$. The average of the measured values was determined $(r=0.93)$ and used in analysis. For abdominal curl-ups, measurements were obtained using the same method. The participants rested for 5 minutes between individual exercises and for 10 seconds between measurements. They rested for 5 minutes after each exercise to reduce muscle fatigue. To exclude the possibility of carryover effect, exercise effect, and effect of order, combinations of exercise postures and exercise were performed in a random order (Fig. 1).

In this study, subjects were asked to cease urination 1 hour prior to the experiment and to drink $600-750 \mathrm{ml}$ of water 30 minutes prior to the experiment ${ }^{18)}$. An educational session was conducted for the abdominal drawing-in maneuver to help participants understand the experimental procedures. Participants were trained to increase the thickness of the transverse abdominis muscle while leaving the external and internal oblique muscles relatively unchanged, achieved with the aid of ultrasound monitoring and pulling the lower abdomen toward the spine at the end of normal expiration ${ }^{14,15)}$. Participants were instructed to pull the internal muscle of the pelvis upward as in the case of patients with urination problems, which resulted in maximum contraction of the pelvic floor muscle. During this process, participants were advised not to allow movement of the pelvis or lumbar spine ${ }^{19}$ ). For training of the participants were asked to bend their knees while in the supine position and to place the soles of their feet flat on the ground. They were then instructed to gaze toward the ceiling and to raise the hips and maintain them in that state for 5 seconds in accordance with a verbal instruction from the measurer, which was "Lift the pelvis". Participants were subsequently asked to bend their knees while in a supine position and to widen both feet to shoulder-width apart, without leaning the legs to either side. After being asked to gaze toward the ceiling, they were instructed to raise and maintain the head and shoulders for 5 seconds in a state instructed by an observer. This was the training provided for abdominal curl-ups.

Regarding the ultrasound equipment, an Achievo CST (V2U Healthcare, Singapore) was used. Measurements were obtained using a 3.5 MHz curved array transducer. The measurement mode was B mode, and the depth was fixed at $130 \mathrm{~mm}$. For training of the abdominal drawing-in maneuver, a transducer was located in the anteromedial direction at the midpoint between the iliac crest and the lowest rib. To evaluate the pelvic floor muscle, the perineal region was assessed to ensure that the lower bladder was visualized while the transducer was located in cross-section of the abdomen and to ensure that the angle between the abdomen and the transducer from about $5 \mathrm{~cm}$ above the pubis symphysis was $15-30^{\circ 10}$.

To measure the movement of the pelvic floor muscle, the movement distance of the lower bladder was measured. A measurement tool was used to calculate the movement distance at the time when the lower edge of the bladder moved due to fascia tension when the pelvic floor muscle contracted. In this study, an X was used to indicate the lower bladder during bridging exercises. Another X was used to mark the movement distance when subjects performed maximal expiration during bridging exercises as well as maximum contraction of the pelvic floor muscle during bridging exercises. In addition, the maximum distance moved by the lower bladder per exercise during bridging exercises was measured. The position of the lower bladder during abdominal curl-ups was marked with an $\mathrm{O}$. The individual movement distances of participants were also marked with Os when they performed the abdominal drawing-in maneuvers during abdominal curl-ups, maximal expiration during abdominal curl-ups, and maximal contraction of the pelvic floor muscle during abdominal curl-ups. In addition, 
the distance that the lower bladder moved per exercise to its maximum elevation during abdominal curl-ups was measured. If elevation of the lower bladder moved upward, the distance it moved was recorded as a negative value. Such an occurrence indicated that the pelvic floor muscle did not contract ${ }^{2,12)}$ (Fig. 2).

For all statistical analyses in this study, PASW Statistics ver. 18.0 was used. Descriptive statistics were obtained for general participant characteristics. To compare movement distance according to the exercise method, repeated measures ANOVA was used. For the post-test, The Bonferroni test was used as a post hoc test. To compare the distance of the lower bladder according to exercise posture, two-way ANOVA was used.

\section{RESULTS}

There were 30 participants in the present study (15 men, 15 women). Baseline characteristics of the participants are shown in Table 1. There were significant differences in the maximal movement distance of the lower bladder between the abdominal drawing-in maneuver, maximal expiration, and pelvic floor muscle maximal contraction during the bridging exercise and abdominal curl-ups $(\mathrm{p}<0.05)$. The maximal movement distance of the lower bladder showed significant differences for both the abdominal drawing-in maneuver and maximal expiration compared with that for pelvic floor muscle maximal contraction. In addition, significant differences was observed for the abdominal drawing-in maneuver compared with the maximal expiration $(\mathrm{p}<0.05)$ (Tables 2 and 3). Comparison of the ultrasound assessments of the abdominal drawing-in maneuver, maximal expiration, and pelvic floor muscle maximum contraction according to exercise posture, the results of the two-way ANOVA showed a significant difference during the bridging exercises and abdominal curl-up according to posture $(\mathrm{F}=$ 21.823, $\mathrm{p}<0.05)$. A significant difference was seen in the movement distance of the lower bladder between the abdominal drawing-in maneuver, maximal expiration, and pelvic floor muscle maximal contraction $(\mathrm{F}=279.272, \mathrm{p}<0.05)$. A significant interaction effect was observed between exercise posture and exercise method $(F=6.220, p<0.05)$. In comparison of ultrasound assessments of the abdominal drawing-in maneuver, maximal expiration, and pelvic floor muscle maximum contraction according to gender of the participant, the results of the two-way ANOVA showed a significant difference during the of bridging exercises and abdominal curl-up according to the exercise method $(\mathrm{F}=431.001, \mathrm{p}<0.05)$. No significant difference was observed according to gender, and there was no significant interaction effect of gender and exercise method.

\section{DISCUSSION}

This study was conducted to compare the effects of the abdominal drawing-in maneuver, maximal expiration, and pelvic floor muscle maximal contraction on the movement of the pelvic floor muscles during bridging exercises and abdominal curl-ups. The results showed abdominal curl-ups and pelvic floor muscle maximal contraction during bridging exercises increased the movement of the pelvic floor muscle, and pelvic floor muscle contraction during abdominal curl-up increased movement of the pelvic floor muscle more than that at the time of bridging exercises.

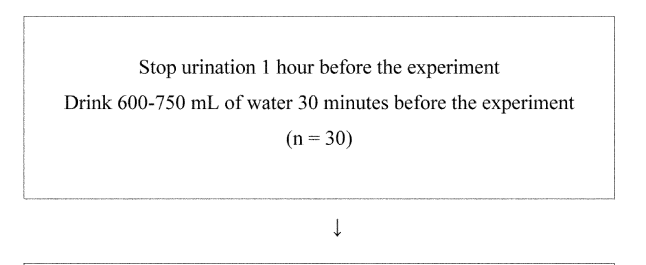

Training on the abdominal drawing-in maneuver and pelvic floor maximal contraction

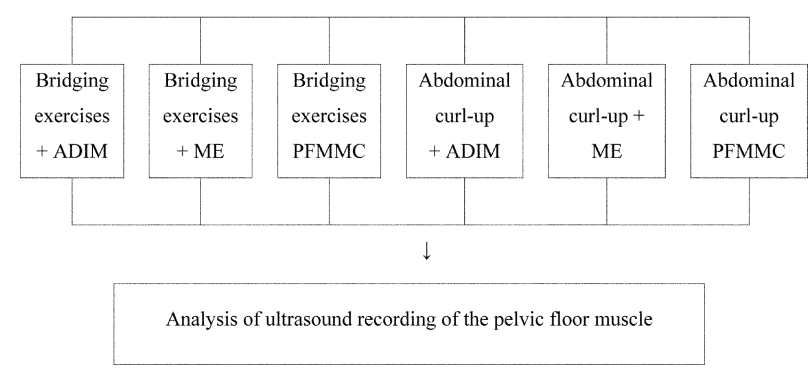

Fig. 1. Experimental procedure ADIM: Abdominal drawing-in maneuver; ME: maximal expiration; PFMMC: pelvic floor muscle maximal contraction

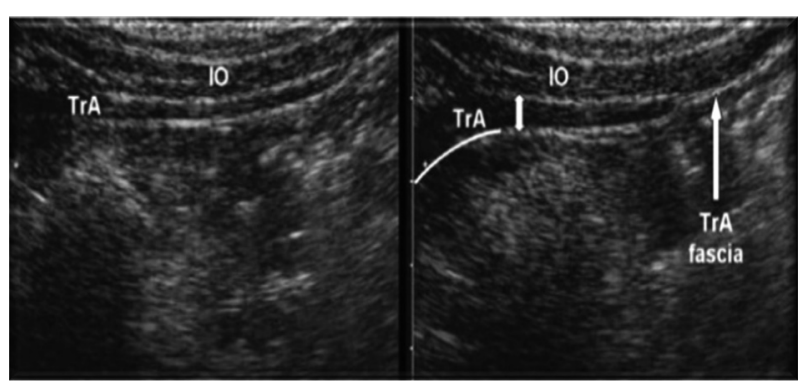

Fig. 2. Abdominal drawing-in maneuver exercise

Table 1. Baseline characteristics of the subjects

\begin{tabular}{lcc}
\hline & $\begin{array}{c}\text { Men } \\
(\mathrm{n}=15)\end{array}$ & $\begin{array}{c}\text { Women } \\
(\mathrm{n}=15)\end{array}$ \\
\hline Age (years) & 28.6 & 29.8 \\
Height $(\mathrm{cm})$ & 175.3 & 162.2 \\
Weight $(\mathrm{kg})$ & 70.9 & 50.1 \\
BMI $\left(\mathrm{kg} / \mathrm{m}^{2}\right)$ & 23.1 & 19.2 \\
\hline
\end{tabular}

BMI: body mass index 
Studies have reported that the pelvic floor muscles control urination and stabilize the lumbopelvic girdle and decreased function of the pelvic floor muscle causes the spine to become unstable, which is closely related to low back pain and pelvic pain ${ }^{1}$. The normalized movement distance of the lower bladder during pelvic floor muscle exercise using ultrasound revealed a distance of $0.27 \mathrm{~mm}$ in women with no back pain but a distance of $0.13 \mathrm{~mm}$ in women with back pain ${ }^{2}$. For men without chronic pelvic pain, the normalized distance using ultrasound was $0.31 \mathrm{~mm}$. However, for men with chronic pelvic pain, the normalized distance using ultrasound was $0.17 \mathrm{~mm}$, representing a decrease of approximately one-half ${ }^{12}$.

Sapsford et al. ${ }^{20)}$ reported that all abdominal muscles have been activated when the pelvic floor muscle contracts maximally, regardless of the posture of the lumbar spine. Neumann and Gill ${ }^{19)}$ reported that the activity of the transverse abdominis muscle reaches $10 \%$ when the pelvic floor muscle contracts maximally, whereas the same activity of the transverse abdominis muscle reaches $94 \%$ and $114 \%$ at the time of maximal pelvic floor muscle contraction and the selective contraction of the transverse abdominis muscle, respectively.

In studies on pelvic floor muscle training, some studies argued that the pelvic floor muscle must be trained with trunk stabilization exercises ${ }^{4}, 21$. Recent studies showed that symptoms of urinary incontinence are mitigated and the strength and endurance of the pelvic floor muscle increase with training. Moreover, these studies have proposed a variety of ways to promote the muscle activity of the deep abdominal muscle. The abdominal drawing-in maneuver, in which the abdomen is pulled up towards the spine at the end of normal expiration, is an exercise to shrink the transverse abdominis muscle independently, reducing muscle activity at the time of bridging exercises and abdominal curl-up, as well as the target action, such as lumbar lordosis ${ }^{22}$.

Otani et al. ${ }^{8)}$ reported that maximal expiration also increased the transverse abdominis and abdominal oblique muscles higher than euthymic-phased expiration and decreased the external oblique muscle. Ishida et al. ${ }^{23)}$ indicated that it is difficult to correctly perform the abdominal drawing-in maneuver and suggested that maximal expiration is another method of activating the deep abdominal muscle. In a comparative study of the abdominal drawing-in maneuver and maximal expiration, it reported that the thickness of the transverse abdominis and external oblique muscles increased by $32.8 \%$ and $14.7 \%$, respectively, and that the activity of the external oblique muscle increased by $15.6 \% \mathrm{MVC}$. In a comparative study of the abdominal drawing-in maneuver and maximal expiration before and after their application, the difference in the transverse abdominis muscle before and after the application of the abdominal drawing-in maneuver and maximal expiration was $1.1 \%$, whereas the difference before and after maximal expiration in the transverse abdominis muscle increased by $5.4 \%{ }^{9}$. This study showed that when participants were instructed to perform the abdominal drawing-in maneuver and maximal contraction of the pelvic floor muscle, the lower bladder was elevated due to the contraction of the pelvic floor muscle, whereas the lower bladder fell during maximal exhalation. The pelvic floor muscle is relaxed and falls during inspiration and contracts and elevates during expiration in association with breathing ${ }^{14)}$. In the abdominal drawing-in maneuver, the lower bladder is elevated due to the indirect contraction of the pelvic floor muscle even though participants were not instructed to directly contract the pelvic floor muscle in the abdominal drawing-in maneuver through the contraction of the transverse abdominis muscle. However, this study suggests that during maximal expiration, the pelvic floor muscle does not contract, as indicated by the contraction of the rectus abdominis muscle and external oblique muscle rather than the contraction of the transverse abdominis muscle.

Table 2. Maximal movement distance of the lower bladder for the abdominal drawing-in maneuver, maximal expiration, and pelvic floor muscle maximal contraction during the bridging exercises

\begin{tabular}{lcccc}
\hline & $\begin{array}{c}\text { Bridging } \\
\text { exercises } \\
+ \text { ADIM }(\mathrm{A})\end{array}$ & $\begin{array}{c}\text { Bridging } \\
\text { exercises } \\
+ \text { ME }(\mathrm{B})\end{array}$ & $\begin{array}{c}\text { Bridging } \\
\text { exercises } \\
+ \text { PFMMC }(\mathrm{C})\end{array}$ & Post hoc \\
\hline Movement distance of the lower bladder $(\mathrm{cm})$ & $0.6 \pm 0.2^{\mathrm{a}}$ & $-0.4 \pm 0.2$ & $0.8 \pm 0.2^{*}$ & A $|\mathrm{B}| \mathrm{C}$ \\
\hline
\end{tabular}

${ }^{\mathrm{a}}$ Mean \pm SD.

ADIM: abdominal drawing-in maneuver; ME: maximal expiration; PFMMC: pelvic floor muscle maximum contraction

Table 3. Comparison of ultrasound assessments of the abdominal drawing-in maneuver, maximal expiration, and pelvic floor muscle maximum contraction during abdominal curl-up

\begin{tabular}{lcccc}
\hline & $\begin{array}{c}\text { Abdominal } \\
\text { curl-up } \\
\text { ADIM } \\
\text { (A) }\end{array}$ & $\begin{array}{c}\text { Abdominal } \\
\text { curl-up } \\
+ \text { ME }\end{array}$ & $\begin{array}{c}\text { Abdominal } \\
\text { curl-up } \\
+ \text { PFMMC } \\
\text { (B) }\end{array}$ & Post hoc \\
\hline Movement distance of the lower bladder $(\mathrm{cm})$ & $0.7 \pm 0.2^{\text {a }}$ & $-0.4 \pm 0.2$ & $1.1 \pm 0.3$ & A | B |C \\
\hline${ }^{a}$ Mean \pm SD. & & & & \\
ADIM: abdominal drawing-in maneuver; ME: maximal expiration; PFMMC: pelvic floor muscle maximum contraction
\end{tabular}


In a study comparing the contraction of the deep abdominal muscles and that of the pelvic floor muscle, Sapsford et al. ${ }^{24)}$ suggested that the application of pelvic floor muscle contraction and abdominal drawing in maneuver. In addition, there was an abdominal muscle training group containing obese women with incontinence who were instructed to contract the transverse abdominis and internal oblique muscles and a voluntary pelvic floor muscle training group. The results showed an increase in pelvic muscle contraction by $15.6 \%$ and $18.0 \%$ after 12 weeks and 24 weeks, respectively, in the abdominal muscle training group, suggesting that training the deep abdominal muscle increases the strength of the pelvic floor muscle more than does voluntary pelvic floor muscle training ${ }^{25)}$. However, it demonstrated that when participants were instructed to perform simultaneous contraction of the pelvic floor muscle and contraction of the transverse abdominis and pelvic floor muscles, the movement distance of the lower bladder, elevated under abdominal ultrasound, suggesting that pelvic floor muscle contraction increased the movement distance of the lower bladder more than the transverse abdominis muscle contraction $^{26)}$. This study found that during pelvic floor muscle training, maximal contraction of the pelvic floor muscle, rather than that of the transverse abdominis muscle, is more effective. This study's results support the results of Bø et al. ${ }^{26)}$ study. In addition, the movement distance of the lower bladder similarly increased in the abdominal drawing-in maneuver during bridging exercises and abdominal curl-ups. In addition, suggesting that abdominal curl-up effectively shrinks the pelvic floor muscle by applying the interventions requiring deep abdominal muscle and pelvic floor muscle contraction using exercises to increase the abdominal muscle activity. Additionally, men showed an increase of $0.10 \mathrm{~cm}$ in the movement distance of the lower bladder at the time of the contraction of the pelvic floor muscle. This is explained by strength differences. There was no significant difference according to gender.

This study demonstrated that an effective method of training the pelvic floor muscles at the time of trunk stabilization exercises should include both the indirect instruction of the contraction of the pelvic floor muscle through the abdominal drawing-in maneuver and the direct instruction of the maximal contraction of the pelvic floor muscle. In the comparison of exercises, abdominal curl-ups improved the movement of the pelvic floor muscle better than did the bridging exercises.

Abdominal ultrasound is non-invasive, safe, and useful for evaluating and visualizing the movement of the pelvic floor muscle. A strength of this method is that it is an easy method since subjects can be assessed while they are clothed. In previous studies, it reported that ultrasound was an objective index for evaluating the functionality and strength of the pelvic floor muscle with MRI as well as a tool for assessing pelvic floor muscle contraction. The reliability of abdominal ultrasound is 0.93 , and it has a high correlation with manual vaginal assessments ${ }^{19)}$ and with perineal ultrasound assessments ${ }^{6,13,25,27)}$.

Limitations of this study are as follows: the sample size was small, and thus the results may not be generalizable. Women's muscles are weaker than those of men; therefore, muscle fatigue may also have an influence; it will be necessary to make a comparison regarding the contraction of the pelvic floor muscle at the time of bridging exercises and abdominal curl-up. In addition, further studies are needed to obtain accurate measurements through electromyography and a vaginal pressure meter to obtain accurate data regarding muscle function of the pelvic floor muscle.

\section{REFERENCES}

1) Sapsford R: Rehabilitation of pelvic floor muscles utilizing trunk stabilization. Man Ther, 2004, 9: 3-12. [Medline] [CrossRef]

2) Arab AM, Behbahani RB, Lorestani L, et al.: Assessment of pelvic floor muscle function in women with and without low back pain using transabdominal ultrasound. Man Ther, 2010, 15: 235-239. [Medline] [CrossRef]

3) Khorasani B, Arab AM, Sedighi Gilani MA, et al.: Transabdominal ultrasound measurement of pelvic floor muscle mobility in men with and without chronic prostatitis/chronic pelvic pain syndrome. Urology, 2012, 80: 673-677. [Medline] [CrossRef]

4) Mohseni-Bandpei MA, Rahmani N, Behtash H, et al.: The effect of pelvic floor muscle exercise on women with chronic non-specific low back pain. J Bodyw Mov Ther, 2011, 15: 75-81. [Medline] [CrossRef]

5) Vera-Garcia FJ, Grenier SG, McGill SM: Abdominal muscle response during curl-ups on both stable and labile surfaces. Phys Ther, 2000, 80: 564-569. [Medline]

6) Stevens VK, Coorevits PL, Bouche KG, et al.: The influence of specific training on trunk muscle recruitment patterns in healthy subjects during stabilization exercises. Man Ther, 2007, 12: 271-279. [Medline] [CrossRef]

7) Teyhen DS, Rieger JL, Westrick RB, et al.: Changes in deep abdominal muscle thickness during common trunkstrengthening exercises using ultrasound imaging. J Orthop Sports Phys Ther, 2008, 38: 596-605. [Medline] [CrossRef]

8) Otani Y, Itotani K, Maeda N, et al.: Reliability of the deep abdominal muscle thickness measurements using ultrasonography in normal subjects. J Phys Ther Sci, 2011, 23: 357-359. [CrossRef]

9) Ishida H, Watanabe S: Changes in lateral abdominal muscles' thickness immediately after the abdominal drawing-in 
maneuver and maximum expiration. J Bodyw Mov Ther, 2013, 17: 254-258. [Medline] [CrossRef]

10) Ariail A, Sears T, Hampton E: Use of transabdominal ultrasound imaging in retraining the pelvic-floor muscles of a woman postpartum. Phys Ther, 2008, 88: 1208-1217. [Medline] [CrossRef]

11) Chehrehrazi M, Arab AM, Karimi N, et al.: Assessment of pelvic floor muscle contraction in stress urinary incontinent women: comparison between transabdominal ultrasound and perineometry. Int Urogynecol J Pelvic Floor Dysfunct, 2009, 20: 1491-1496. [Medline] [CrossRef]

12) Sherburn M, Murphy CA, Carroll S, et al.: Investigation of transabdominal real-time ultrasound to visualise the muscles of the pelvic floor. Aust J Physiother, 2005, 51: 167-170. [Medline] [CrossRef]

13) Arab AM, Behbahani RB, Lorestani L, et al.: Correlation of digital palpation and transabdominal ultrasound for assessment of pelvic floor muscle contraction. J Manual Manip Ther, 2009, 17: e75-e79. [Medline] [CrossRef]

14) Thompson JA, O'Sullivan PB, Briffa K, et al.: Assessment of pelvic floor movement using transabdominal and transperineal ultrasound. Int Urogynecol J Pelvic Floor Dysfunct, 2005, 16: 285-292. [Medline] [CrossRef]

15) Henry SM, Westervelt KC: The use of real-time ultrasound feedback in teaching abdominal hollowing exercises to healthy subjects. J Orthop Sports Phys Ther, 2005, 35: 338-345. [Medline] [CrossRef]

16) Teyhen DS, Miltenberger CE, Deiters HM, et al.: The use of ultrasound imaging of the abdominal drawing-in maneuver in subjects with low back pain. J Orthop Sports Phys Ther, 2005, 35: 346-355. [Medline] [CrossRef]

17) Kim BI, Hwang-Bo G, Kim HR: Comparison of abdominal muscle thickness with vaginal pressure changes in healthy women. J Phys Ther Sci, 2014, 26: 427-430. [Medline] [CrossRef]

18) Price N, Dawood R, Jackson SR: Pelvic floor exercise for urinary incontinence: a systematic literature review. Maturitas, 2010, 67: 309-315. [Medline] [CrossRef]

19) Neumann P, Gill V: Pelvic floor and abdominal muscle interaction: EMG activity and intra-abdominal pressure. Int Urogynecol J Pelvic Floor Dysfunct, 2002, 13: 125-132. [Medline] [CrossRef]

20) Sapsford RR, Hodges PW, Richardson CA, et al.: Co-activation of the abdominal and pelvic floor muscles during voluntary exercises. Neurourol Urodyn, 2001, 20: 31-42. [Medline] [CrossRef]

21) Hung HC, Hsiao SM, Chih SY, et al.: Effect of pelvic-floor muscle strengthening on bladder neck mobility: a clinical trial. Phys Ther, 2011, 91: 1030-1038. [Medline] [CrossRef]

22) Teyhen DS, Bluemle LN, Dolbeer JA, et al.: Changes in lateral abdominal muscle thickness during the abdominal drawing-in maneuver in those with lumbopelvic pain. J Orthop Sports Phys Ther, 2009, 39: 791-798. [Medline] [CrossRef]

23) Ishida $\mathrm{H}$, Hirose $\mathrm{R}$, Watanabe $\mathrm{S}$ : Comparison of changes in the contraction of the lateral abdominal muscles between the abdominal drawing-in maneuver and breathe held at the maximum expiratory level. Man Ther, 2012, 17: 427-431. [Medline] [CrossRef]

24) Sapsford RR, Clarke B, Hodges PW: The effect of abdominal and pelvic floor muscle activation patterns on urethral pressure. World J Urol, 2013, 31: 639-644. [Medline] [CrossRef]

25) Kamel DM, Thabet AA, Tantawy SA, et al.: Effect of abdominal versus pelvic floor muscle exercises in obese Egyptian women with mild stress urinary incontinence: A randomised controlled trial. Hong Kong Physiotherapy J, 2013, 31: 12-18. [CrossRef]

26) Bø K, Sherburn M, Allen T: Transabdominal ultrasound measurement of pelvic floor muscle activity when activated directly or via a transversus abdominis muscle contraction. Neurourol Urodyn, 2003, 22: 582-588. [Medline] [CrossRef]

27) Bø K, Sherburn M: Evaluation of female pelvic-floor muscle function and strength. Phys Ther, 2005, 85: 269-282. [Medline] 